\title{
Robust Adaptive Sliding Mode Consensus of Multiagent Systems with Perturbed Communications and Actuators
}

\author{
Xiao-Zheng Jin and Zhong-Hu Yuan \\ The Key Laboratory of Manufacturing Industrial Integrated Automation, Shenyang University, Shenyang, Liaoning 110044, China \\ Correspondence should be addressed to Xiao-Zheng Jin; jin445118@163.com
}

Received 10 January 2013; Accepted 8 March 2013

Academic Editor: Yang Tang

Copyright (C) 2013 X.-Z. Jin and Z.-H. Yuan. This is an open access article distributed under the Creative Commons Attribution License, which permits unrestricted use, distribution, and reproduction in any medium, provided the original work is properly cited.

\begin{abstract}
This paper deals with the asymptotic consensus problem for a class of multiagent systems with time-varying additive actuator faults and perturbed communications. The $L_{2}$ performance of systems is also considered in the consensus controller designs. The upper and lower bounds of faults and perturbations in actuators and communications and controller gains are assumed to be unknown but can be estimated by designing some indirect adaptive laws. Based on the information from the adaptive estimation mechanism, the distributed robust adaptive sliding mode controllers are constructed to automatically compensate for the effects of faults and perturbations and to achieve any given level of $L_{2}$ gain attenuation from external disturbance to consensus errors. Through Lyapunov functions and adaptive schemes, the asymptotic consensus of resulting adaptive multiagent system can be achieved with a specified performance criterion in the presence of perturbed communications and actuators. The effectiveness of the proposed design is illustrated via a decoupled longitudinal model of F-18 aircraft.
\end{abstract}

\section{Introduction}

The consensus behavior of multiagent systems has received significant attention over the last few years. It involves in lots of practical control systems and many physical phenomenon, such as synchronization of coupled oscillators [1], rendezvous in space [2], aircraft formation control [3], and flocking theory [4]. Recently, lots of researchers have focused on the development of methodologies to solve consensus problem with some usual issues existed in communications such as time-delays $[5,6]$, perturbations [7-15], and faulty communication links [16-18].

As we know, state consensus may not be guaranteed under the influence of network perturbations. In [7], the bounded tracking results were obtained for multiagent consensus with an active leader in the presence of bounded perturbations. A stochastic model for distributed average consensus with zero mean noise was presented in [8]. Recently, the authors of [9] utilized an adaptive method to deal with a class of known upper bound disturbances. In addition, from the Laplacian eigenvalue standpoint, paper [10] focused on maximizing the second smallest eigenvalue of a state-dependent graph
Laplacian to improve the robustness of the dynamic systems. The recent paper [11] gave a lazy consensus protocol against unknown but bounded disturbances and achieved bounded consensus results. Note that the aforementioned systems cannot guarantee asymptotic consensus when the disturbance always exists in the systems. Therefore, the capability of disturbance rejection for the previous systems seems weak. Recently, an asymptotic consensus result has been obtained in [12] under a matching condition in the presence of timedelays and disturbances in communications. But the design is complicated and the performances of systems are not considered in the paper.

It is well known that actuators play an important role in the consensus of multiagent systems. Many researchers were devoted to the study of fault-tolerant control against actuator/sensor faults to ensure the stability and performance optimization of systems (e.g., see [19-21]). Paper [17] considered consensus problem with missing data in actuators and Markovian communication failure, and the communication failure process was reduced to a Bernoulli process. The recent paper [22] studied fault diagnosis for a class of discrete time-delayed complex interconnected networks with linear 
coupling in the case of actuator faults. The authors of [23] analyzed the performances of a team of unmanned vehicles with some actuator fault types, such that loss of effectiveness and lock-in-place. In [18], the bias actuators have been considered in synchronization of master-slave systems using an indirect adaptive method. Here, the similar additive actuator faults which can also be considered as perturbed actuators are treated by using an adaptive sidling mode method.

It should be noted that the system performances, such as $H_{\infty}, H_{2}, L_{2}$ performances, are rarely considered in the consensus designs. In [13], the $H_{\infty}$ consensus control problem was considered in directed networks of delayed and nondelayed agents using a matrix inequality method. The $H_{\infty}$ consensus filtering problem was dealt with in [14] by a difference linear matrix inequalities over a finite-horizon for sensor networks with multiple missing measurements. The authors of [15] utilized a model transformation approach and matrix theory to solve the $H_{\infty}$ consensus for secondorder multiagent systems with multiple asymmetric timevarying delays. Different from those papers using matrix inequality methods, this paper mainly considers the studies of $L_{2}$ performance of multiagent systems by an adaptive method.

In this paper, we consider the consensus problem of multiagent systems in the presence of perturbed communications and actuators with a specified performance criterion. Here, the bounds of additive faults and the size of perturbations in communications are not necessary to be known. Based on the Lyapunov stability theory, a novel adaptive sliding mode control strategy is developed to achieve asymptotic consensus of the multiagent systems. On the basis of this proposed method, an integral sliding manifold is developed for average consensus and varying consensus. Some adaptive schemes are proposed to estimate the bounds of faults and perturbations and controller gains. Then, adaptive sliding mode controllers are constructed relying on the updated gains. By using the designed controller, the faulty and perturbed factors effects can be completely compensated and the asymptotic consensus can be achieved in the finite time with any given level of $L_{2}$ gain attenuation. Besides, it should be noted that the new proposed adaptive design method is not necessary for the estimations to give the exact information.

The asymptotic consensus problem formulation is described in Section 2. In Section 3, the adaptive sidling mode state feedback controllers are developed. Section 4 gives an example and simulation. Finally, conclusions are given in Section 5.

\section{Preliminaries and Problem Statement}

In this paper, we consider a multiagent system $G$ composed of $N$ interconnected linear time-invariant continuous time agents $G_{i}, i=1,2, \ldots, N$, which can be illustrated as an undirected graph. Each edge $\left(v_{j}, v_{i}\right)$ corresponds to an available information link from agent $i$ to agent $j$. Then, the
$N$ nodes constitute a network as the following state-space equation:

$$
\begin{array}{r}
\dot{x}_{i}(t)=A x_{i}(t)+\sum_{j=1, j \neq i}^{N} a_{i j} A_{12}\left(x_{j}(t)-x_{i}(t)+d_{i j}(t)\right) \\
+B_{2}\left(u_{i}(t)+f_{i}(t)\right)+B_{1} w_{i}(t),
\end{array}
$$

where $x_{i}(t) \in R^{n}$ is the state of node $i$, and $u_{i}(t) \in R^{q}$ is the control input; $a_{i j} \in R$ represents the topological structure of the network, which is an element of Laplacian matrix [24] satisfying $a_{i i}=-\sum_{j=1, j \neq i}^{N} a_{i j}$, and $A_{12} \in R^{n \times n}$ is the inner coupling matrix describing the interconnections among components; $d_{i j}(t) \in R^{n}$ is the network perturbation in communication between the $i$ agent and the $j$ agent, which is satisfied by $\underline{d}_{i j} \leq d_{i j} \leq \bar{d}_{i j}$, where $\underline{d}_{i j}$ and $\bar{d}_{i j}$ denote the unknown amplitude size of $d_{i j} ; f_{i}(t) \in R^{q}$ denotes the additive actuator faults. Here, we assume that $f_{i}(t)$ can be described by a nonlinear function and bounded by unknown lower and upper bounds $f_{i}$ and $\bar{f}_{i} ; w_{i}(t) \in R^{m}$ is a continuous vector function denoting external disturbance and possible variations with respect to the nominal parameter values for the system; $A, B_{1}$, and $B_{2}$ are real constant matrices with appropriate dimensions.

Then, by the Kronecker product, the multiagent system (1) can be rewritten as

$$
\begin{aligned}
\dot{x}(t)= & \left(I \otimes A+L \otimes A_{12}\right) x(t)+\left(I \otimes A_{12}\right) d(t) \\
& +\left(I \otimes B_{2}\right) u(t)+\left(I \otimes B_{2}\right) f(t)+\left(I \otimes B_{1}\right) w(t),
\end{aligned}
$$

where $x=\left(x_{1}^{T}, \ldots, x_{N}^{T}\right)^{T}, u=\left(u_{1}^{T}, \ldots, u_{N}^{T}\right)^{T}, f=$ $\left(f_{1}^{T}, \ldots, f_{N}^{T}\right)^{T}, w=\left(w_{1}^{T}, \ldots, w_{N}^{T}\right)^{T}, d=\left(d_{1}^{T}, \ldots, d_{N}^{T}\right)^{T}, d_{i}=$ $\sum_{j=1, j \neq i}^{N} a_{i j} d_{i j}, i=1,2, \ldots, N$, and $L$ is called the graph Laplacian induced by the information flow $G$ and is defined by

$$
l_{i j}= \begin{cases}-\sum_{k=1, k \neq i}^{N} a_{i k}, & j \neq i, \\ a_{i j}, & j=i .\end{cases}
$$

Similar to [12], to ensure the achievement of average consensus objective, the following assumption in consensus design is also assumed to be valid.

Assumption 1. For multiagent system (2) and any appropriate dimension matrix $K_{1}$, there exist matrix functions $H, Z$ of appropriate dimensions such that

$$
A_{12}+B_{2} K_{1}=B_{2} H, \quad A=B_{2} Z,
$$

respectively.

Define

$$
\alpha=\frac{1}{N} \sum_{i=1}^{N} x_{i}(0),
$$

where $x_{i}(0), i=1,2, \ldots, N$, are the known initial values of system states. Then, for the sake of solving the consensus 
problems of multiagent systems in the presence of external faults, the local consensus protocol is considered as follows:

$$
\begin{array}{r}
u_{i}(t)=-K_{1}\left[\sum_{j=1, j \neq i}^{N} a_{i j}\left(x_{i}(t)-x_{j}(t)+d_{i j}\right)\right. \\
\left.+c_{i}\left(x_{i}(t)-\alpha\right)\right]+v_{i}(t),
\end{array}
$$

where $i, j=1,2, \ldots, N, a_{i j}$ is defined as in (1), $c_{i}$ is a positive constant, $K_{1}$ is the control gain, and $v_{i}(t)$ is an adaptive control function which will be designed in later.

Then, by the Kronecker product, (6) can be rewritten as

$$
\begin{aligned}
u(t)= & -K_{1}(L+C) \otimes I x(t) \\
& +K_{1}\left(C \mathbf{1}_{N}\right) \otimes \alpha+\left(I \otimes K_{1}\right) d+v,
\end{aligned}
$$

where $u=\left(u_{1}^{T}, \ldots, u_{N}^{T}\right)^{T}, v=\left(v_{1}^{T}, \ldots, v_{N}^{T}\right)^{T}$, and $C$ is an $N \times N$ diagonal matrix whose $i$ th diagonal element is $c_{i}$, while $\mathbf{1}_{N}$ represents $[1,1, \ldots, 1]_{N}^{T}$.

Then, substituting (7) into (2), the closed-loop system model is stated as

$$
\begin{aligned}
\dot{x}(t)= & \left(I \otimes A+L \otimes A_{12}\right) x(t) \\
& -\left(I \otimes B_{2}\right) K_{1}(L+C) \otimes I x(t) \\
& +\left(I \otimes B_{2}\right) K_{1}\left(C \mathbf{1}_{N}\right) \otimes \alpha \\
& +\left(I \otimes\left(A_{12}+B_{2} K_{1}\right)\right) d(t) \\
& +\left(I \otimes B_{2}\right) f(t)+\left(I \otimes B_{2}\right) v(t) \\
& +\left(I \otimes B_{1}\right) w(t) .
\end{aligned}
$$

Now, let

$$
\bar{x}(t)=x(t)-\mathbf{1}_{N} \alpha .
$$

Due to the characteristic of Laplacian matrix $L$, see (3), we have

$$
\begin{aligned}
\left(I \otimes B_{2}\right) K_{1}(L+C) \otimes I \bar{x}(t)= & \left(I \otimes B_{2}\right) K_{1}(L+C) \otimes I x(t) \\
& -\left(I \otimes B_{2}\right) K_{1}\left(C \mathbf{1}_{N}\right) \otimes \alpha(t) .
\end{aligned}
$$

Then, following (10) and substituting (9) into (8), the closedloop system can be rewritten as

$$
\begin{aligned}
\dot{\bar{x}}(t)= & \left(I \otimes A+L \otimes A_{12}-\left(I \otimes B_{2}\right) K_{1}(L+C) \otimes I\right) \bar{x}(t) \\
& +\left(I \otimes B_{2}\right) v(t)+\left(I \otimes\left(A_{12}+B_{2} K_{1}\right)\right) d(t) \\
& +\left(I \otimes B_{2}\right) f(t)+(I \otimes A) \alpha+\left(I \otimes B_{1}\right) w(t) .
\end{aligned}
$$

According to Assumption 1, we have

$$
\begin{aligned}
\dot{\bar{x}}(t)= & \left(I \otimes A+L \otimes A_{12}-\left(I \otimes B_{2}\right) K_{1}(L+C) \otimes I\right) \bar{x}(t) \\
& +\left(I \otimes B_{2}\right) v(t)+\left(I \otimes B_{2}\right) e(t)+\left(I \otimes B_{1}\right) w(t),
\end{aligned}
$$

where $e=\left(e_{1}^{T}, \ldots, e_{N}^{T}\right)^{T}, e_{i}(t)=f_{i}(t)+H d_{i}(t)+Z \alpha$. Since $f_{i}(t), d_{i}(t)$, and $\alpha$ are bounded signals, we know that $e_{i}$ is also a bounded signal and denote $e_{i}$ and $\bar{e}_{i}$ as unknown larger constants than lower and upper bounds of $e_{i}$, respectively.

Then, the objective of this paper is to make sure that system (12) is asymptotically stable; namely,

$$
\lim _{t \rightarrow \infty} \bar{x}(t)=0,
$$

that is,

$$
\lim _{t \rightarrow \infty} x(t)=\mathbf{1}_{N} \alpha
$$

with any given $L_{2}$ performance index under the influence of perturbed communications and actuators.

\section{Main Results}

In this section, we develop the adaptive laws to estimate unknown controller gains for designing robust adaptive sliding mode controllers to eliminate the effects of communication perturbations and actuator faults and, simultaneously, to achieve any given $L_{2}$ performance criterion of the closedloop system (12).

The composite sliding surface for the closed-loop system (12) is chosen as

$$
s(\bar{x}(t))=0,
$$

with $s(\bar{x}(t)) \equiv:\left(s_{1}\left(\bar{x}_{1}(t)\right), s_{2}\left(\bar{x}_{2}(t)\right), \ldots, s_{N}\left(\bar{x}_{N}(t)\right)\right)^{T}$ and

$$
\begin{gathered}
s_{i}\left(\bar{x}_{i}(t)\right)=\bar{x}_{i}(t)-\bar{x}_{i}\left(t_{0}\right) \\
-\int_{t_{0}}^{t}\left[A \bar{x}_{i}(\tau)+\sum_{j=1, j \neq i}^{N} a_{i j} A_{12}\left(\bar{x}_{j}(\tau)-\bar{x}_{i}(\tau)\right)\right. \\
-B_{2} K_{1}\left(\sum_{j=1, j \neq i}^{N} a_{i j}\left(\bar{x}_{j}(\tau)-\bar{x}_{i}(\tau)\right)\right. \\
\left.\left.+c_{i} \bar{x}_{i}(\tau)\right)\right] d \tau
\end{gathered}
$$

where $K_{1}$ is the controller gain proposed in (6) which is obtained by solving the following linear matrix inequality:

$$
\begin{aligned}
& \left(I \otimes A+L \otimes A_{12}-B_{2} K_{1}(L+C) \otimes I\right)^{T}(I \otimes P) \\
& \quad+(I \otimes P)\left(I \otimes A+L \otimes A_{12}-B_{2} K_{1}(L+C) \otimes I<0\right.
\end{aligned}
$$

where $P$ is a positive definite matrix. Note that the matrix $K_{1}$ is designed such that the nominal fault-free system (12) is stable and some prescribed specifications would also be satisfied via this nominal state feedback control. Here, the term of $\bar{x}_{i}\left(t_{0}\right)$ achieves the nice property that $\sigma_{i}\left(\bar{x}_{i}\left(t_{0}\right)\right)=0$ such that the reaching phase is eliminated. 
Now, consider the controller model (6) with controller gain $K_{1}$ solved by (17). We design control function $v_{i}(t)$ as follows:

$$
\begin{aligned}
v_{i}= & \widehat{\phi}_{i}\left\|s_{i}^{T} P B_{2}\right\| \operatorname{sgn}\left(s_{i}^{T} P B_{2}\right)^{T} \\
& +K_{2}\left[\left(I-\rho_{i}\right) \widehat{\bar{e}}_{i}(t)+\rho_{i} \widehat{\hat{e}}_{i}(t)\right],
\end{aligned}
$$

where $\phi_{i}$ is an existed but unknown large enough positive constant satisfying

$$
2 \phi_{i}\left\|s_{i}^{T} P B_{2}\right\|^{2} \geq\left\|s_{i}^{T} P B_{1}\right\|^{2}+\gamma_{i}\left\|s_{i}\right\|^{2}
$$

where $\gamma_{i}$ is any given $L_{2}$ performance index, and $\widehat{\phi}_{i}$ is the estimation of $\phi_{i}$ updated by the following adaptive laws:

$$
\frac{d \widehat{\phi}_{i}(t)}{d t}=r_{i}\left\|s_{i}^{T} P B_{2}\right\|^{2},
$$

where $r_{i}>0$ is the weight of adaptive law $\widehat{\phi}_{i}(t)$. The sign function $\operatorname{sgn}\left(s_{i}^{T} P B_{2}\right)^{T}=\left[\operatorname{sgn}\left(b_{i 1}\right), \ldots, \operatorname{sgn}\left(b_{i q}\right)\right]^{T}$, where $b_{i l}$, $l=1,2, \ldots, q$, is the $l$ element of the vector $s_{i}^{T} P B_{2}$ and $\operatorname{sgn}\left(b_{i l}\right)$ defined by

$$
\operatorname{sgn}\left(b_{i l}\right)= \begin{cases}-1, & \text { if } b_{i l}>0, \\ 1, & \text { if } b_{i l}<0, \\ 0, & \text { if } b_{i l}=0,\end{cases}
$$

and $P$ is a positive symmetric matrix designed in (17); $\rho_{i}$ is the switching factor defined between constants 0 and 1 defined by

$$
\begin{aligned}
& \rho_{i}=\operatorname{diag}\left[\rho_{i 1}, \rho_{i 2}, \ldots, \rho_{i q}\right], \\
& \rho_{i l}= \begin{cases}0, & b_{i l} \geq 0, \\
1, & b_{i l}<0 .\end{cases}
\end{aligned}
$$

$\widehat{\bar{e}}_{i l}(t)$ and $\hat{e}_{i l}(t)$ are the estimations of $\bar{e}_{i l}$ and $\underline{e}_{i l}$, respectively, updated by the following adaptive laws:

$$
\begin{aligned}
& \frac{d \hat{\bar{e}}_{i l}(t)}{d t}=t_{i l} b_{i l}, \\
& \frac{d \widehat{e}_{i l}(t)}{d t}=t_{i l} b_{i l},
\end{aligned}
$$

where $t_{i l}>0$ are the adaptive law gains to be designed according to practical application.

Let

$$
\begin{aligned}
& \tilde{\phi}_{i}(t)=\widehat{\phi}_{i}(t)-\phi_{i}, \\
& \tilde{\bar{e}}_{i}(t)=\widehat{\bar{e}}_{i}(t)-\bar{e}_{i}, \\
& \tilde{e}_{i}(t)=\underline{e}_{i}(t)-\underline{e}_{i},
\end{aligned}
$$

where $\bar{e}_{i}=\left[\bar{e}_{i 1}, \bar{e}_{i 2}, \ldots, \bar{e}_{i n}\right]^{T}, \underline{e}_{i}=\left[\underline{e}_{i 1}, \underline{e}_{i 2}, \ldots, \underline{e}_{i n}\right]^{T}, i=$ $1,2, \ldots, N$.
Because to $\phi_{i}, \bar{e}_{i}, \underline{e}_{i}$ are constants, the error system can be written as the following equations:

$$
\dot{\widetilde{\phi}}_{i}(t)=\dot{\widehat{\phi}}_{i}(t), \quad \dot{\overline{\bar{e}}}_{i}(t)=\dot{\overline{\bar{e}}}_{i}(t), \quad \dot{\tilde{e}}_{i}(t)=\dot{\hat{\hat{e}}}_{i}(t) .
$$

Thus, for the multiagent system described by (12), we propose the adaptive robust local control scheme (7) with the control gain function $K_{2}$ given by

$$
K_{2}=-I
$$

Hence, the following theorem can be obtained, which shows the uniform ultimate boundedness of the closed-loop system (12) and the error system (25).

Theorem 2. Consider the closed-loop multiagent system described by (12) satisfying Assumption 1. By using the control scheme $u(t)$ described in (7) with adaptive laws (20) and (23) and control gain functions (26), one can guarantee that all closed-loop system signals are bounded and $\lim _{t \rightarrow \infty} x_{i}(t)=\alpha$ with any given $L_{2}$ performance index $\gamma_{i}$ for any initial value $x\left(t_{0}\right)$, if there exists a symmetric matrix $P>0$ in (17).

Proof. For the adaptive robust closed-loop system described by (12), we first define a Lyapunov functional candidate as

$$
\begin{aligned}
V(t)= & s^{T}(I \otimes P) s+\sum_{i=1}^{N} \frac{\widetilde{\phi}_{i}^{2}}{r_{i}} \\
& +\sum_{i=1}^{N} \sum_{l=1}^{n} \frac{\left(1-\rho_{i l}\right) \widetilde{\bar{e}}_{i l}^{2}}{t_{i l}}+\sum_{i=1}^{N} \sum_{l=1}^{n} \frac{\rho_{i l} \widetilde{e}_{i l}^{2}}{t_{i l}} .
\end{aligned}
$$

From (12) and (16), the derivative of $s(\bar{x}(t))$ with respect to time can be calculated as follows:

$$
\begin{aligned}
\dot{s}(\bar{x}(t)) & \\
= & \dot{\bar{x}}(t)-\dot{\bar{x}}\left(t_{0}\right) \\
& -\left(I \otimes A+L \otimes A_{12}-K_{1}(L+C) \otimes I\right) \bar{x}(t) \\
= & \left(I \otimes B_{2}\right)\left(\operatorname{sgn}\left(s^{T} \widehat{\phi}_{s} \otimes P B_{2}\right)^{T}\right) \\
& +\left(I \otimes B_{2}\right)\left(I \otimes K_{2}\right)[(I \otimes(I-\rho)) \hat{\bar{e}}(t)+(I \otimes \rho) \underline{\hat{e}}(t)] \\
& +\left(I \otimes B_{2}\right) e(t)+\left(I \otimes B_{1}\right) w(t),
\end{aligned}
$$

where $\hat{\bar{e}}=\left(\hat{\bar{e}}_{1}^{T}, \hat{\bar{e}}_{2}^{T}, \ldots, \hat{\bar{e}}_{N}^{T}\right)^{T}, \underline{\hat{e}}=\left(\underline{\hat{e}}_{1}^{T}, \underline{\hat{e}}_{2}^{T}, \ldots, \underline{\hat{e}}_{N}^{T}\right)^{T}, \rho=$ $\operatorname{diag}\left[\rho_{1}, \rho_{2}, \ldots, \rho_{N}\right], \widehat{\phi}_{s}=\operatorname{diag}\left[\widehat{\phi}_{1}\left\|s_{1}^{T} P B_{2}\right\|, \widehat{\phi}_{2}\left\|s_{2}^{T} P B_{2}\right\|, \ldots\right.$, $\left.\widehat{\phi}_{N}\left\|s_{N}^{T} P B_{2}\right\|\right]$. 
Then, following Assumption 1, the time derivative of $V(t)$ for $t>0$ can be described as

$$
\begin{aligned}
& \dot{V}(t)=+2 s^{T}(I \otimes P)\left(I \otimes B_{2}\right)\left(\operatorname{sgn}\left(s^{T} \widehat{\phi}_{s} \otimes P B_{2}\right)^{T}\right) \\
& +2 s^{T}(I \otimes P)\left(I \otimes B_{2}\right) e+2 s^{T}(I \otimes P)\left(I \otimes B_{1}\right) w \\
& +2 s^{T}(I \otimes P)\left(I \otimes B_{2}\right)\left(I \otimes K_{2}\right)(\hat{\bar{e}}+\rho \underline{\hat{e}}-\rho \hat{\bar{e}}) \\
& +\sum_{i=1}^{N} \frac{2 \widetilde{\phi}_{i} \dot{\bar{\phi}}_{i}}{r_{i}}+\sum_{i=1}^{N} \sum_{l=1}^{n} \frac{2\left(1-\rho_{i l}\right) \widetilde{\bar{e}}_{i l} \dot{\overline{\bar{e}}}_{i l}}{t_{i l}} \\
& +\sum_{i=1}^{N} \sum_{l=1}^{q} \frac{2 \rho_{i l} \underline{\tilde{e}}_{i l} \dot{\tilde{\tilde{e}}}_{i l}}{t_{i l}} \\
& =2 \sum_{i=1}^{N} \widehat{\phi}_{i}\left\|s_{i}^{T} P B_{2}\right\| s_{i}^{T} P B_{2} \operatorname{sgn}\left(s_{i}^{T} P B_{2}\right)^{T} \\
& +2 \sum_{i=1}^{N} s_{i}^{T} P B_{1} w_{i}+2 s^{T}(I \otimes P) \sum_{i=1}^{N} \sum_{l=1}^{q} B_{i} e_{i l} \\
& +2 s^{T}(I \otimes P) \sum_{i=1}^{N} \sum_{l=1}^{q} B_{i} k_{2 l}\left(\widehat{\bar{e}}_{i l}+\rho_{i l} \widehat{e}_{i l}-\rho_{i l} \widehat{\bar{e}}_{i l}\right) \\
& +\sum_{i=1}^{N} \frac{2 \widetilde{\phi}_{i} \dot{\tilde{\phi}}_{i}}{r_{i}}+\sum_{i=1}^{N} \sum_{l=1}^{q} \frac{2\left(1-\rho_{i l}\right) \tilde{\bar{e}}_{i l} \dot{\bar{e}}_{i l}}{t_{i l}} \\
& +\sum_{i=1}^{N} \sum_{l=1}^{q} \frac{2 \rho_{i l} \underline{\tilde{e}}_{i l} \dot{\tilde{\underline{e}}}_{i l}}{t_{i l}}
\end{aligned}
$$

where $B_{i}$ is the $i$ th column of $I \otimes B_{2}, i=1,2, \ldots, N$.

Note that

$$
\begin{aligned}
& s^{T}(I \otimes P) \sum_{i=1}^{N} \sum_{l=1}^{q} B_{i} e_{i l} \\
& \quad \leq s^{T}(I \otimes P) \sum_{i=1}^{N} \sum_{l=1}^{q} B_{i}\left(\bar{e}_{i l}+\rho_{i l} e_{i l}-\rho_{i l} \bar{e}_{i l}\right),
\end{aligned}
$$

where $\rho_{i l}$ are denoted in (22). By the adaptive laws chosen in (23) and controller gain function chosen in (26), then (29) can be rewritten as

$$
\begin{aligned}
\dot{V}(t) \leq & 2 \sum_{i=1}^{N} \widehat{\phi}_{i}\left\|s_{i}^{T} P B_{2}\right\| s_{i}^{T} P B_{2} \operatorname{sgn}\left(s_{i}^{T} P B_{2}\right)^{T} \\
& +2 \sum_{i=1}^{N} s_{i}^{T} P B_{1} w_{i}+2 s^{T}(I \otimes P) \\
& \times \sum_{i=1}^{N} \sum_{l=1}^{q} B_{i}\left(\bar{e}_{i l}+\rho_{i l} \underline{e}_{i l}-\rho_{i l} \bar{e}_{i l}\right)+2 s^{T}(I \otimes P)
\end{aligned}
$$

$$
\begin{aligned}
& \times \sum_{i=1}^{N} \sum_{l=1}^{q} B_{i} k_{2 l}\left(\hat{\bar{e}}_{i l}+\rho_{i l} \widehat{\underline{e}}_{i l}-\rho_{i l} \widehat{\bar{e}}_{i l}\right) \\
& +\sum_{i=1}^{N} \frac{2 \widetilde{\phi}_{i} \dot{\bar{\phi}}_{i}}{r_{i}}+\sum_{i=1}^{N} \sum_{l=1}^{q} \frac{2\left(1-\rho_{i l}\right) \widetilde{\bar{e}}_{i l} \dot{\overline{\bar{e}}}_{i l}}{t_{i l}} \\
& +\sum_{i=1}^{N} \sum_{l=1}^{q} \frac{2 \rho_{i l} \tilde{\widetilde{e}}_{i l} \dot{\tilde{\vec{e}}}_{i l}}{t_{i l}} \\
& \leq 2 \sum_{i=1}^{N} \widehat{\phi}_{i}\left\|s_{i}^{T} P B_{2}\right\| s_{i}^{T} P B_{2} \operatorname{sgn}\left(s_{i}^{T} P B_{2}\right)^{T} \\
& +2 \sum_{i=1}^{N}\left\|s_{i}^{T} P B_{1}\right\|\left\|w_{i}\right\|+\sum_{i=1}^{N} \frac{2 \widetilde{\phi}_{i} \dot{\tilde{\phi}}_{i}}{r_{i}}
\end{aligned}
$$

From the fact that $s_{i}^{T} P B_{2} \operatorname{sgn}\left(s_{i}^{T} P B_{2}\right)^{T} \leq-\left\|s_{i}^{T} P B_{2}\right\|$ and the adaptive law proposed in (20) with inequality (19), then we have

$$
\begin{aligned}
\dot{V}(t) \leq & -2 \sum_{i=1}^{N} \widehat{\phi}_{i}\left\|s_{i}^{T} P B_{2}\right\|^{2} \\
& +2 \sum_{i=1}^{N}\left\|s_{i}^{T} P B_{1}\right\|\left\|w_{i}\right\|+\sum_{i=1}^{N} \frac{2 \widetilde{\phi}_{i} \dot{\tilde{\phi}}_{i}}{r_{i}} \\
\leq & -\sum_{i=1}^{N}\left(2 \widehat{\phi}_{i}\left\|s_{i}^{T} P B_{2}\right\|^{2}-\left\|s_{i}^{T} P B_{1}\right\|^{2}-\gamma_{i}\left\|s_{i}\right\|^{2}\right) \\
& +\sum_{i=1}^{N}\left(\left\|w_{i}\right\|^{2}-\gamma_{i}\left\|s_{i}\right\|^{2}\right)+\sum_{i=1}^{N} \frac{2 \widetilde{\phi}_{i} \dot{\bar{\phi}}_{i}}{r_{i}} \\
= & \sum_{i=1}^{N}\left(\left\|w_{i}\right\|^{2}-\gamma_{i}\left\|s_{i}\right\|^{2}\right) .
\end{aligned}
$$

Obviously, we have $\dot{V}(t)<0$ if $\left\|s_{i}\right\|>\left\|w_{i}\right\| / \sqrt{\gamma_{i}}$. It means that the sliding manifold signal is uniformly ultimately bounded by $\lim _{t \rightarrow \infty}\left\|s_{i}\right\| \in\left\{s_{i} \in R^{n} \mid\left\|s_{i}\right\| \geq\left\|w_{i}\right\| / \sqrt{\gamma_{i}}\right\}$.

When inequality (32) is integrated over the interval $\left[t_{0}, t\right]$, we obtain

$$
\begin{aligned}
& V(t)-V\left(t_{0}\right) \\
& \leq \sum_{i=1}^{N}\left(-\int_{t_{0}}^{t} \gamma_{i}\left\|s_{i}(\tau)\right\|^{2} d(\tau)+\int_{t_{0}}^{t}\left\|w_{i}(\tau)\right\|^{2} d(\tau)\right) .
\end{aligned}
$$

Then, we have

$$
\sum_{i=1}^{N} \int_{t_{0}}^{t} \gamma_{i}\left\|s_{i}(\tau)\right\|^{2} d(\tau) \leq V\left(t_{0}\right)+\sum_{i=1}^{N} \int_{t_{0}}^{t}\left\|w_{i}(\tau)\right\|^{2} d(\tau)
$$

where $V\left(t_{0}\right)=s\left(t_{0}\right)^{T}(I \otimes P) s\left(t_{0}\right)+\sum_{i=1}^{N}\left(\widetilde{\phi}_{i}^{2}\left(t_{0}\right) / r_{i}\right)+$ $\sum_{i=1}^{N} \sum_{l=1}^{n}\left(\left(1-\rho_{i l}\right) \widetilde{\bar{e}}_{i l}^{2}\left(t_{0}\right) / t_{i l}\right)+\sum_{i=1}^{N} \sum_{l=1}^{n}\left(\rho_{i l} \underline{\tilde{e}}_{i l}^{2}\left(t_{0}\right) / t_{i l}\right)$. 
If the initial conditions are chosen to be zero, then the $L_{2}$ gain becomes clear such that

$$
\begin{aligned}
\gamma_{i} \int_{t_{0}}^{t}\left\|s_{i}(\tau)\right\|^{2} d(\tau) \leq & \int_{t_{0}}^{t}\left\|w_{i}(\tau)\right\|^{2} d(\tau)+\frac{\widetilde{\phi}_{i}^{2}\left(t_{0}\right)}{r_{i}} \\
& +\sum_{l=1}^{n} \frac{\left(1-\rho_{i l}\right) \widetilde{\bar{e}}_{i l}^{2}\left(t_{0}\right)}{t_{i l}}+\sum_{l=1}^{n} \frac{\rho_{i l} \underline{\tilde{e}}_{i l}^{2}\left(t_{0}\right)}{t_{i l}} .
\end{aligned}
$$

Hence, it is easy to see that $\dot{V}(t)<0$ for any $\bar{x} \neq 0$. Thus, the solutions of closed-loop system are uniformly bounded, and the error $\bar{x}(t)$ converges asymptotically to zero. Moreover, from (35) and the results of [25], the $L_{2}$ gain level of the disturbance attenuation can be guaranteed to be a given small value by adjusting $\gamma_{i}$.

Remark 3. The first part of control function $v_{i}(t)$ in (18) is designed for formulating the $L_{2}$ performance index $\gamma_{i}$. The second part of $v_{i}(t)$ is constructed for eliminating the effects of the matched perturbations of actuators and communications. It should be noted that the method largely simplifies the designs in [12].

The average consensus problem has been solved in Theorem 2. Actually, the system can also track the timevarying object by the proposed method. Here, we define that $\alpha(t) \in R^{n}$ is the given command of states in time $t$ for the agents. Without loss of generality, we assume that the command is a differentiable continuous signal which satisfies

$$
\dot{\alpha}(t)=C g(t),
$$

where $g(t)$ is bounded by an unknown positive constant $g_{0}$ such that $\|g(t)\| \leq g_{0}$, and $C$ is a real constant matrix with appropriate dimensions and satisfies the condition $C=B_{2} M$.

Without considering $w_{i}(t)$, then the closed-loop system (12) can be rewritten as

$$
\begin{aligned}
\dot{\bar{x}}(t)= & \left(I \otimes A+L \otimes A_{12}-\left(I \otimes B_{2}\right) K_{1}(L+C) \otimes I\right) \bar{x}(t) \\
& +\left(I \otimes B_{2}\right) v(t)+\left(I \otimes B_{2}\right) e(t),
\end{aligned}
$$

where $e=\left(e_{1}^{T}, \ldots, e_{N}^{T}\right)^{T}, e_{i}(t)=f_{i}(t)+H d_{i}(t)+M g(t)+Z \alpha(t)$. Since $f_{i}(t), d_{i}(t), g(t)$, and $\alpha(t)$ are bounded signals, we know that $e_{i}$ is still a bounded signal.

Then, the following corollary is proposed to obtain asymptotic consensus results of perturbed communications and actuators.

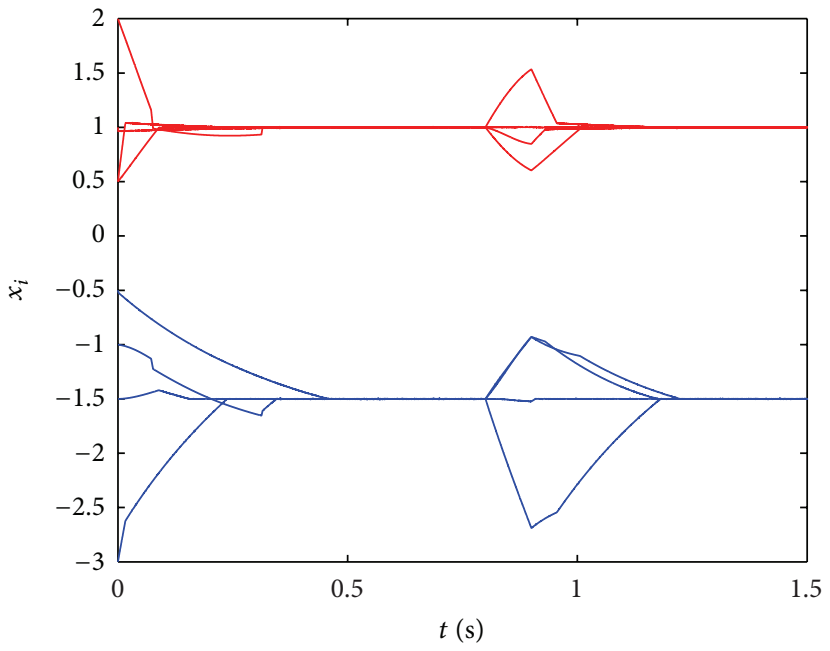

Figure 1: Response curves of the system states $x_{i}(t), i=1,2,3,4$.

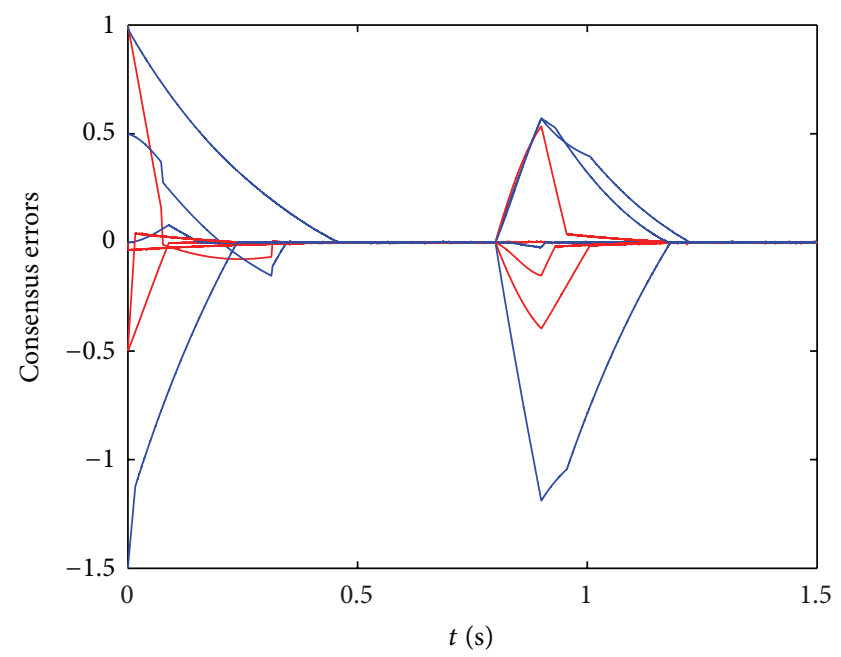

FigURE 2: Response curves of the consensus errors $\bar{x}_{i}(t), i=1,2,3,4$.

Corollary 4. Suppose that Assumption 1 holds. Consider the closed-loop multiagent system described by (37). Then, by using the control scheme $u(t)$ described by

$$
\begin{array}{r}
u_{i}(t)=-K_{1}\left[\sum_{j=1, j \neq i}^{N} a_{i j}\left(x_{i}(t)-x_{j}(t)+d_{i j}\right)\right. \\
\left.+c_{i}\left(x_{i}(t)-\alpha\right)\right] \\
+K_{2}\left[\left(I-\rho_{i}\right) \hat{\bar{e}}_{i}(t)+\rho_{i} \hat{\underline{e}}_{i}(t)\right]
\end{array}
$$

with adaptive laws (23) and control gain functions (26), one can guarantee that all closed-loop multiagent system signals are bounded and $\lim _{t \rightarrow \infty} x_{i}(t)=\alpha(t)$ for any initial value $x(0)$, if there exists a symmetric matrix $P>0$ in (17). 


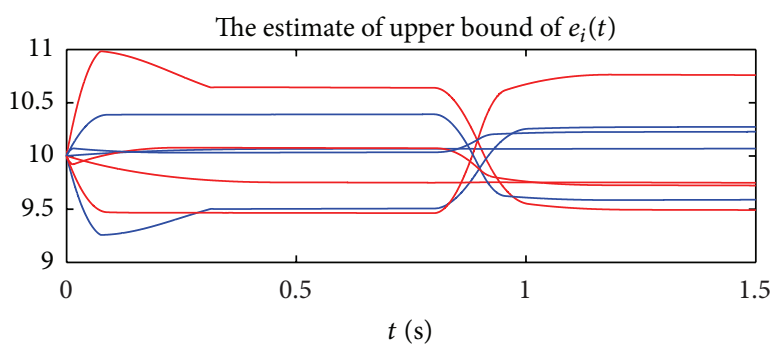

(a)

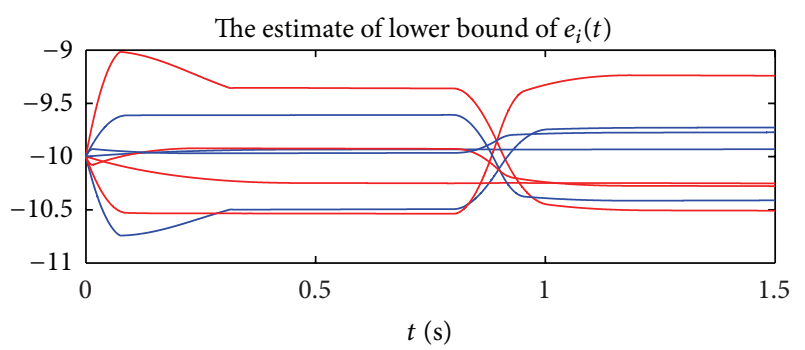

(b)

FIGURE 3: Response curves of the estimate of upper bound and lower bound of $e_{i}(t), i=1,2,3,4$.

Proof. Similar to the proof of Theorem 2 and according to (30), we have $s^{T}(I \otimes P) \sum_{i=1}^{N} \sum_{l=1}^{q} B_{i} h_{l} e_{i l}=s^{T}(I \otimes$ P) $\sum_{i=1}^{N} \sum_{l=1}^{q} B_{i} h_{l}\left(\bar{e}_{i l}+\rho_{i l} e_{i l}-\rho_{i l} \bar{e}_{i l}\right)$ if and only if $s_{i}=0$. Thus, it is obvious that $\dot{V}=0$ if and only if $s_{i}=0, i=1,2, \ldots, N$, and a set $E$ can be found as $E=\{(s, \underline{\widehat{e}}, \hat{\bar{e}}): \dot{V}=0\}=\{(s, \underline{\widehat{e}}, \hat{\bar{e}})$ : $\left.s_{i}=0\right\}$, where $s=\left(s_{1}, s_{2}, \ldots, s_{N}\right)^{T}, \underline{\hat{e}}=\left(\underline{\hat{e}}_{1}, \underline{\hat{e}}_{2}, \ldots, \underline{\hat{e}}_{N}\right)^{T}$, and $\hat{\bar{e}}=\left(\hat{\bar{e}}_{1}, \hat{\bar{e}}_{2}, \ldots, \hat{\bar{e}}_{N}\right)^{T}$. Starting with arbitrary initial values $s_{i}(0), \underline{\hat{e}}_{i}(0)$, and $\hat{\bar{e}}_{i}(0)$, the orbit converges asymptotically to $s_{i}=0, \underline{\underline{e}}_{i}=\underline{e}_{i}$, and $\hat{\bar{e}}_{i}=\bar{e}_{i}$, where $\underline{e}_{i}, \bar{e}_{i}$ are constants. Based on LaSalle invariance principle, the system trajectories converge to the largest positively invariant subset $M=\{(s, \underline{\hat{e}}, \widehat{\bar{e}}): s=$ $0, \underline{\dot{\hat{e}}}=0, \dot{\overline{\bar{e}}}=0\}$. Moreover, according to (12) and (28), we know that the error $s_{i}(t)$ converges asymptotically to zero if there exist $K_{1}$ and $P$ making the inequality (17) hold true.

\section{Numerical Example}

In this section, an example of robust consensus control system design is given to demonstrate the proposed method. A multiagent system is composed of four dynamical agents, which have the same system matrices as follows:

$$
\begin{array}{ll}
A=\left[\begin{array}{cc}
-1 & 0.2 \\
1 & 0.1
\end{array}\right], & B_{2}=\left[\begin{array}{cc}
2 & -1.5 \\
1 & -1
\end{array}\right], \\
A_{12}=\left[\begin{array}{cc}
0.4 & 0 \\
0 & 0.4
\end{array}\right], & B_{1}=\left[\begin{array}{cc}
1.5 & 1 \\
-2 & -1
\end{array}\right],
\end{array}
$$

and the topological structure matrix $L$ in (3) described by

$$
L=\left[\begin{array}{cccc}
-1 & 0.2 & 0.5 & 0.3 \\
0.1 & -1 & 0.6 & 0.3 \\
0.2 & 0.5 & -1 & 0.3 \\
0.1 & 0.3 & 0.6 & -1
\end{array}\right]
$$

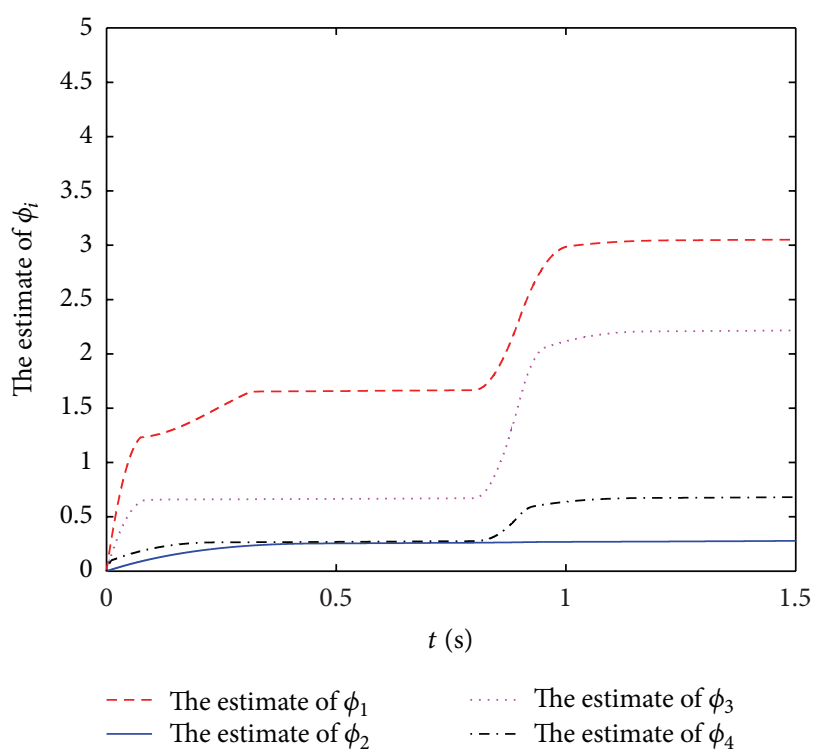

FIGURE 4: Response curves of the estimate of $\phi_{i}, i=1,2,3,4$.

To verify the effectiveness of the proposed adaptive sliding mode method, the simulations are given with the following parameters and initial conditions:

$$
\begin{gathered}
r_{i}=100, \quad t_{i l}=100, \quad \hat{\bar{e}}_{i l}(0)=10, \\
\hat{\underline{e}}_{i l}(0)=-10, \quad c_{i}=1, \\
x_{1}(0)=[2,-1]^{T}, \quad x_{2}(0)=[1,-0.5]^{T}, \\
x_{3}(0)=[0.5,-1.5]^{T}, \quad x_{4}(0)=[0.5,-3]^{T}, \\
i=1,2,3,4, \quad l=1,2 .
\end{gathered}
$$

Then, following the system state initial values, we get the average value $\alpha=[1,-1.5]^{T}$.

Then, solving LMI (17), we can obtain

$$
P=\left[\begin{array}{cc}
0.1260 & -0.0156 \\
-0.0156 & 0.0262
\end{array}\right], \quad K_{1}=\left[\begin{array}{cc}
1.8286 & -2.7429 \\
1.8286 & -3.6571
\end{array}\right] \text {. }
$$

The following case is considered in the simulations; that is, the communication perturbations $d_{12}(t)=$ 


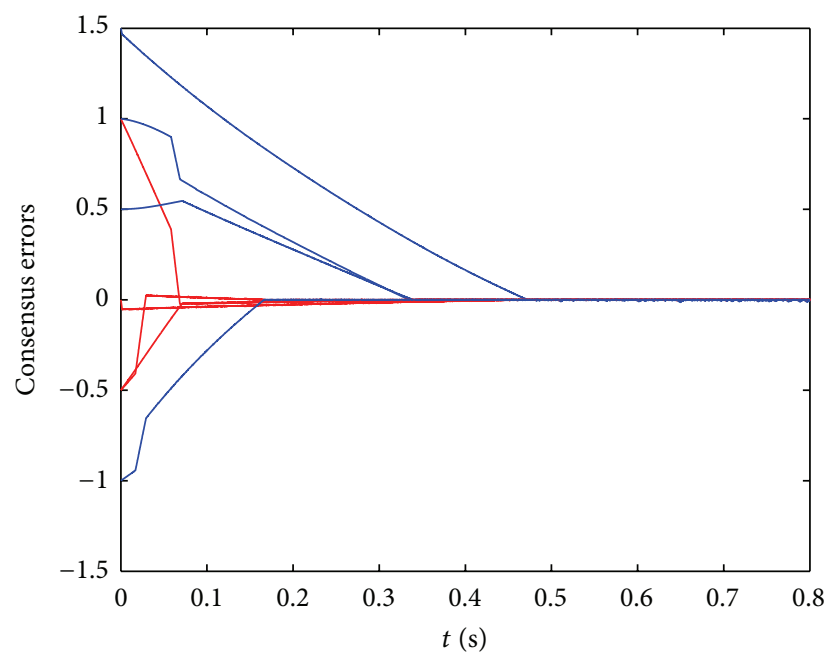

FIGURE 5: Response curves of the consensus errors $\bar{x}_{i}(t)$ with varying target; $i=1,2,3,4$.

$[-0.5,0.2 \sin (2 t)]^{T}, \quad d_{23}(t)=[0.4 \sin (0.2 t),-0.5]^{T}$, $d_{34}(t)=[0.5,0.5 \cos (2 t)]^{T}$, and $d_{41}(t)=[-0.2,0.3 \sin (20 t)]^{T}$ and the additive actuator faults $f_{1}(t)=[0.5,0.5 \cos (0.5 t)]^{T}$, $f_{2}(t)=[-0.5,0.4 \sin (20 t)]^{T}, f_{3}(t)=[0.5,-0.4 \sin (2 t)]^{T}$, and $f_{4}(t)=[0.5 \sin (t),-1.5]^{T}$ enter into the systems at the beginning $(t \geq 0)$. Moreover, the external disturbances $w_{1}(t)=[-5,2 \sin (2 t)]^{T}, w 2(t)=[4 \sin (0.2 t),-5]^{T}$, $w_{3}(t)=[5,5 \cos (2 t)]^{T}$, and $w_{4}(t)=[-2,3 \sin (20 t)]^{T}$ enter into the systems in $t \in[0.8,0.9]$.

Figures 1 and 2 are, respectively, the response curves of the system's states and consensus errors with adaptive robust feedback control scheme in aforementioned case. Figure 3 describes the estimate of upper and lower bounds of perturbations $\hat{\bar{e}}_{i}$ and $\hat{\underline{e}}_{i}, i=1,2,3,4$, respectively. Figure 4 is the response curves of the estimate of controller gain $\phi_{i}, i=$ $1,2,3,4$. It is easy to see that the estimations can convergence but not converge to theirs true values. In our adaptive robust fault-tolerant control design, there is no need for the estimate of $\widehat{\bar{e}}_{i}, \underline{\hat{e}}_{i}, \widehat{\phi}_{i}$ to converge to their true values.

Furthermore, the proposed method can also deal with the time-varying consensus problem. Here, we consider the previous system with the same initial conditions and perturbations and faults, but without considering the effects of external disturbances. The consensus target is described by $\dot{\alpha}(t)=0.5 B_{2}[\sin (t) \cos (t)]^{T}$ with initial values $[1,-1.5]^{T}$. Then, Figure 5 illustrates the response curves of the consensus errors with adaptive sidling mode control scheme (38). The results indicated that the proposed method can also solve the time-varying consensus problem with some matched condition.

\section{Conclusions}

In this paper, we have shown an adaptive design method to solve the robust asymptotic consensus problem for a class of multiagent systems with perturbed communications and faulted actuators. For the sake of automatically compensating for the effects of faults and networked perturbations and specifying $L_{2}$ performance criterion, the consensus protocol is constructed with the adaptive schemes, which are based on the updated adaptation laws to estimate the unknown bounds of perturbations and controller gains online. On the basis of Lyapunov stability theory, it has been shown that the resulting adaptive closed-loop multiagent system can be guaranteed to be asymptotic average and varying consensus with any given $L_{2}$ performance index even in the presence of imperfect communications and actuators. A numerical example has been given to illustrate the effectiveness of the proposed method.

\section{Acknowledgments}

This work is supported by the National Nature Science Foundation (Grant nos. 61104029, 61273155, and 61203087), the Science and Technology Plan of Liaoning province (2011219011), the Natural Science Foundation of Liaoning Province (Grant no. 201202156), and the Scientific Research Foundation for Doctor of Liaoning Province (Grant no. 20121040).

\section{References}

[1] V. M. Preciado and G. C. Verghese, "Synchronization in generalized Erd os-Renye networks of nonlinear oscillators," in Proceedings of the 44th Conference on Decision and Control, and the European Control Conference (CDC-ECC '05), pp. 46284633, Seville, Spain, December 2005.

[2] J. Lin, A. S. Morse, and B. D. O. Anderson, "The multi-agent rendezvous problem," in Proceedings of the 42nd Conference on Decision and Control, pp. 1508-1513, December 2003.

[3] R. Olfati-Saber and R. M. Murray, "Graph rigidity and distributed formation stabilization of multi-vehicle systems," in Proceedings of the Conference on Decision and Control, pp. 29652971, December 2002.

[4] R. Olfati-Saber, "Flocking for multi-agent dynamic systems: algorithms and theory," IEEE Transactions on Automatic Control, vol. 51, no. 3, pp. 401-420, 2006.

[5] R. Olfati-Saber and R. M. Murray, "Consensus problems in networks of agents with switching topology and time-delays," IEEE Transactions on Automatic Control, vol. 49, no. 9, pp. 15201533, 2004.

[6] F. Xiao and L. Wang, "Consensus protocols for discrete-time multi-agent systems with time-varying delays," Automatica, vol. 44, no. 10, pp. 2577-2582, 2008.

[7] Y. Hong, L. Gao, D. Cheng, and J. Hu, "Tracking control for multi-agent consensus with an active leader and variable topology," Automatica, vol. 42, no. 7, pp. 1177-1182, 2006.

[8] W. Yu, G. Chen, Z. Wang, and W. Yang, "Distributed consensus filtering in sensor networks," IEEE Transactions on Systems, Man, and Cybernetics B, vol. 39, no. 6, pp. 1568-1577, 2009.

[9] Z. G. Hou, L. Cheng, and M. Tan, "Decentralized robust adaptive control for the multiagent system consensus problem using neural networks," IEEE Transactions on Systems, Man, and Cybernetics B, vol. 39, no. 3, pp. 636-647, 2009. 
[10] Y. Kim and M. Mesbahi, "On maximizing the second smallest eigenvalue of a state-dependent graph Laplacian," IEEE Transactions on Automatic Control, vol. 51, no. 1, pp. 116-120, 2006.

[11] D. Bauso, L. Giarré, and R. Pesenti, "Lazy consensus for networks with unknown but bounded disturbances," in Proceedings of the 46th IEEE Conference on Decision and Control (CDC '07), pp. 2283-2288, New Orleans, La, USA, December 2007.

[12] X. Z. Jin and G. H. Yang, "Distributed robust adaptive control for a class of dynamical complex networks against imperfect communications," International Journal of Systems Science, vol. 42, no. 3, pp. 457-468, 2011.

[13] P. Lin, Y. Jia, and L. Li, "Distributed robust $H_{\infty}$ consensus control in directed networks of agents with time-delay," Systems and Control Letters, vol. 57, no. 8, pp. 643-653, 2008.

[14] B. Shen, Z. Wang, and Y. S. Hung, "Distributed $H_{\infty}$-consensus filtering in sensor networks with multiple missing measurements: the finite-horizon case," Automatica, vol. 46, no. 10, pp. 1682-1688, 2010.

[15] Y. Sun and L. Wang, " $H_{\infty}$ consensus of second-order multiagent systems with asymmetric delays," Systems \& Control Letters, vol. 61, no. 8, pp. 857-862, 2012.

[16] S. Kar and J. M. F. Moura, "Distributed consensus algorithms in sensor networks with imperfect communication: link failures and channel noise," IEEE Transactions on Signal Processing, vol. 57, no. 1, pp. 355-369, 2009.

[17] Y. Hu, J. Lam, and J. Liang, "Consensus control of multi-agent systems with missing data in actuators and Markovian communication failure," International Journal of Systems Science, 2012.

[18] X. Z. Jin, G. H. Yang, and W. W. Che, "Adaptive synchronization of master- slave large-scale systems against bias actuators and network attenuations," International Journal of Control, Automation and Systems, vol. 10, no. 6, pp. 1102-1110, 2012.

[19] Q. Hu and B. Xiao, "Adaptive fault tolerant control using integral sliding mode strategy with application to flexible spacecraft," International Journal of Systems Science, 2013.

[20] X. Z. Jin and G. H. Yang, "Robust adaptive fault-tolerant compensation control with actuator failures and bounded disturbances," Acta Automatica Sinica, vol. 35, no. 3, pp. 305309, 2009.

[21] X. Z. Jin and G. H. Yang, "Robust fault-tolerant $H_{\infty}$ control with adaptive com- pensation," Acta Automatica Sinica, vol. 39, no. 1, pp. 31-42, 2013.

[22] Z. Wang, H. Guan, and C. Zheng, "Fault diagnosis observer design for discrete- time delayed complex interconnected networks with linear coupling," Mathematical Problems in Engineering, vol. 2012, Article ID 860489, 22 pages, 2012.

[23] E. Semsar-Kazerooni and K. Khorasani, "Team consensus for a network of unmanned vehicles in presence of actuator faults," IEEE Transactions on Control Systems Technology, vol. 18, no. 5, pp. 1155-1161, 2010.

[24] C. Godsil and G. Royle, Algebraic Graph Theory, Springer, New York, NY, USA, 2001.

[25] A. Schaft and A. J. Schaft, $L_{2}$ Gain and Passivity Techniques in Nonlinear Control, Springer, London, UK, 1999. 


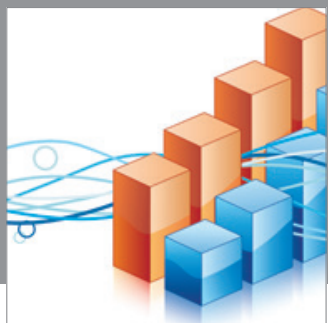

Advances in

Operations Research

mansans

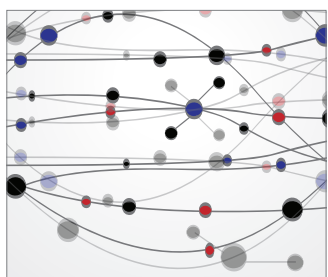

The Scientific World Journal
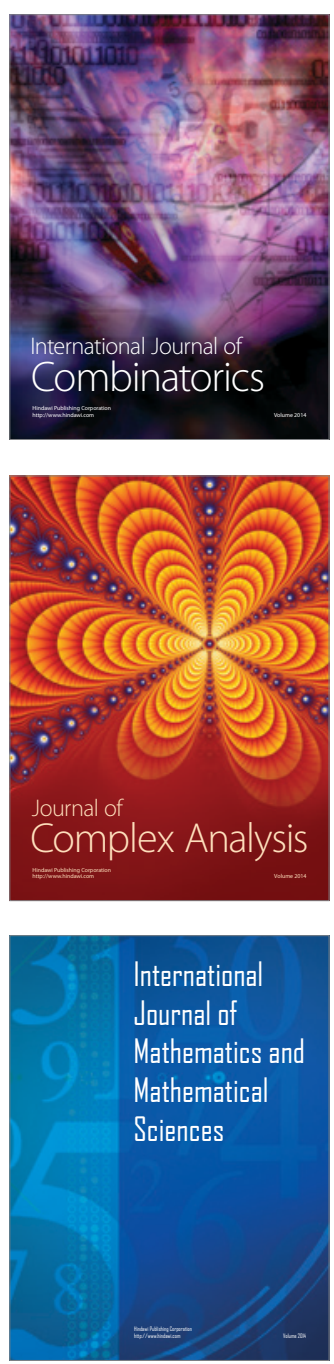
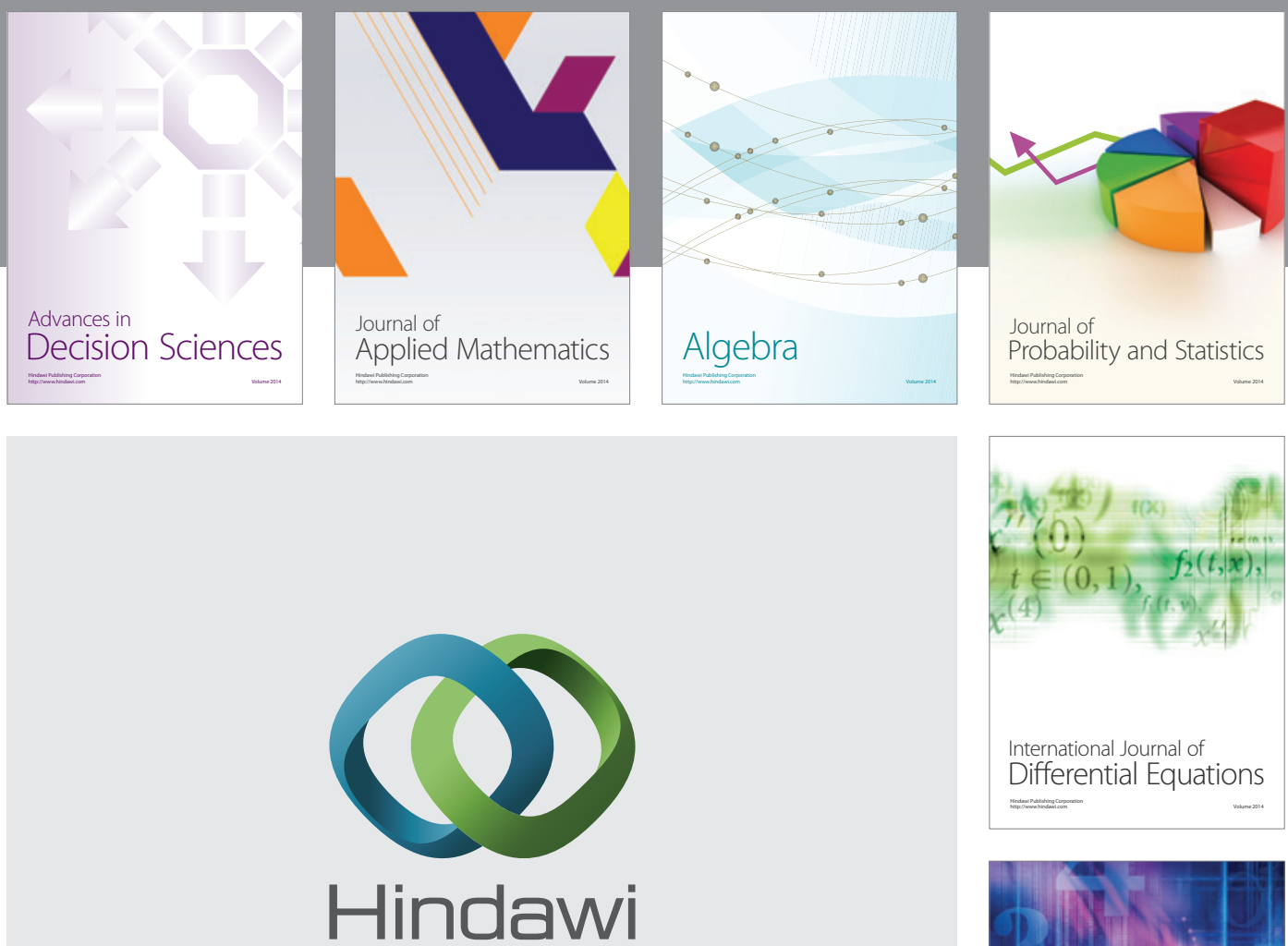

Submit your manuscripts at http://www.hindawi.com
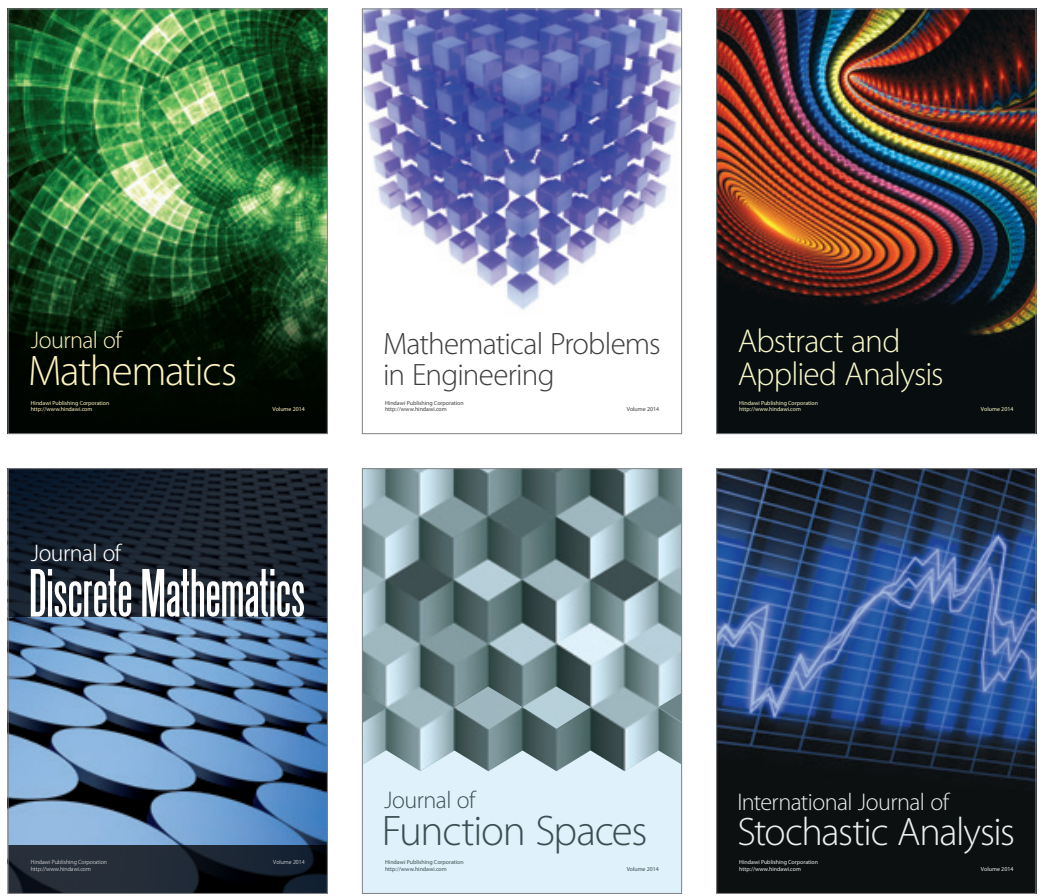

Journal of

Function Spaces

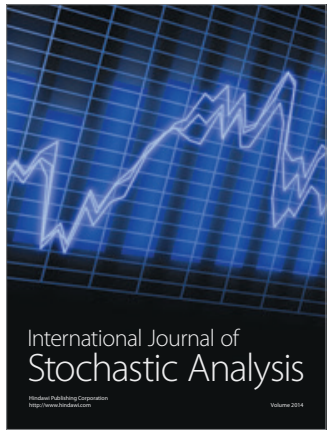

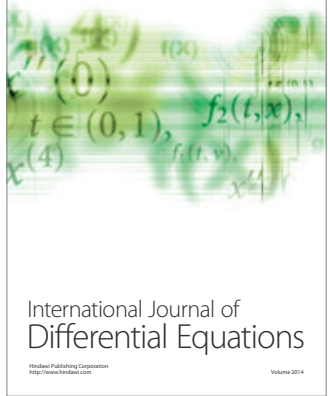
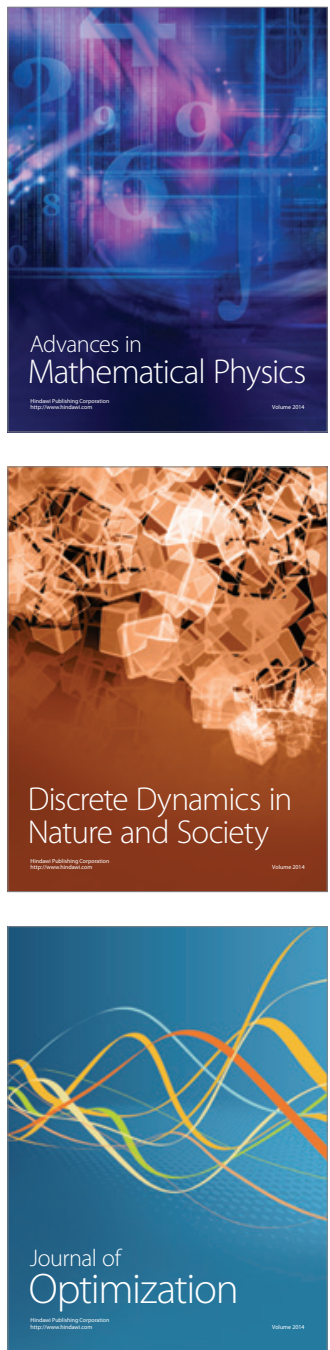